\title{
The whole process cost management based on BIM
}

\author{
jixue Zou, jia Guo, jing Wu
}

No.8, dongyi road, chang 'an district, xi 'an city, shaanxi province China

Building 10-13, building no.58, south second ring, xi 'an city China

277 yantaxi road, xi 'an city, shaanxi province China

894912195@qq.com

Keywords: The project cost ,BIM , Management

Abstract. The whole process cost management is to ensure that the investment benefit of construction projects, from feasibility study on construction project started by the preliminary design, expand the preliminary design, construction design, contracting, construction, commissioning, completion, production and final accounts, after the evaluation of the overall process, all around the engineering cost of business behavior and organizational activities.This paper introduces how the cost management of BIM technology plays a role in the project construction.

\section{Introduction}

At present, China's construction project is in the whole process cost management mode. Engineering cost control is divided into decision-making stage, implementation stage (design, construction) and operation stage according to the stage of engineering formation. Corresponding to it is estimation, budget estimate, contract price, construction budget, payment of progress payment, completion settlement, etc.

Engineering cost management every link data volume is quite large, the calculation procedure is also very complicated. With the development of market economy, the large scale complex projects in large and medium-sized cities have also increased significantly, and the cost management has become more and more difficult.The traditional manual calculation and the software budget of the single machine have fallen far behind the needs of The Times.There has been no breakthrough in cost management software.The present cost management technology mainly has the following limitations:

(1) the cost analysis data is not accurate enough, the function is relatively weak.

The cost of the current mainstream software method to the set price or form, can only analyze a list of the total data, data granularity far cannot achieve project management process requirements, also can only meet the bidding budget and settlement, cannot satisfy the floor according to the construction area according to component analysis, cannot achieve more based on the analysis of the time dimension.

(2) Cost is difficult to achieve process management.Detailed cost management needs to be detailed to different time, different components, different processes and so on. The construction enterprise only knows the end of the project at one end of the price,The construction enterprise only knows the price of the project beginning and ending,the process cost management completely abandoned.It was only after the project was done that the actual cost is largely different from the budget, and it was too late to take action.In the case of developer, budget overexpenditure phenomenon is very common. The important reason for cost overruns is the inability to make accurate estimates, followed by the lack of reliable cost data

Building Information model (Building Information Modeling BIM), namely the implementation of construction engineering design, construction and management of engineering Information models and parameterization, BIM also has been described by many domestic and foreign designers 
as the second "revolutionary" technology in the construction industry after CAD technology [1].The following is based on BIM technology cost management how to play a role in various stages of project construction.

\section{Decision making stage}

In the stage of project investment decision, the data of the previous BIM model can be utilized [2], and the cost of similar projects per square meter is estimated, and the cost of such a project is estimated.

According to the historical engineering model of BIM library, make simple adjustment, estimate the total investment of the project and improve the accuracy

\section{The design phase}

We can use the historical data of the BIM model to design the quota, so that the economic and rationality of the design can be guaranteed.

The design fee of the project is only $1 \%$ to $3 \%$ of the cost of the project, but the design determines more than $70 \%$ of the cost of the project. The design stage is the key to control the cost of the project.

The design quota indicator is proposed by the developer independently. The aim of the current quota design is to change the project cost from "control" to "reduce" project cost.

The BIM model is used to measure the cost data. On the one hand, it can improve the accuracy of calculation, and on the other hand, it can improve the measurement depth.

After the design is completed, the BIM model is used to quickly make the budget estimate, and check whether the design indicators meet the requirements, control the total investment, and give play to the value of the limit design

\section{Bidding stage}

With the gradual application of the bill of quantities bidding in the domestic construction market, the developer can provide the quantities required for the bidding quickly and accurately according to the BIM model.

Avoid disputes arising from engineering quantity problems during construction.

For builder, because the bidding time is tight, to calculate by hand, most projects are hard to verify the list of quantities, for only a part of the project, some items check, unavoidably appear error.

The BIM model can be used to quickly check the engineering quantity, so as to avoid the loss of project due to the problem of quantity, similar to the case of China railway construction.

The calculation of engineering is the basis of the compilation of the project budget, but the calculation process is very tedious and boring, and the cost engineer can easily cause the calculation error for the cause and affect the accuracy of the subsequent calculation.

It is reasonable for the general project personnel to calculate the engineering quantity error in the case of plus or minus 3\%. If a large project is encountered, the result of complex engineering and irregular engineering is more difficult to say.

\section{The construction phase}

In the tender after completion, and determine the total package party led by developer design company, supervision unit to participate in one of the biggest range of design disclosure and drawing review meeting, though, it was conducted in the tender is completed,the design most can only increase the project cost to resolve the problem, but can be settled before the formal construction, can reduce visa, reduce rework cost and the contractor's construction claim, and with 
the intervention of the contractor and supervision company, can review from the Angle of construction and supervision of the drawings, find errors and unreasonable factors.

However, the traditional drawing review is based on two dimensional plane drawings, and each professional drawing is designed separately. It is difficult to detect problems by artificial inspection.

Using BIM technology, we can integrate the majors into the unified platform and carry out three-dimensional collision inspection. We can find a large number of design mistakes and unreasonable places to provide effective support for project cost management.,

of course, Collision check is not only used in the construction stage, the blue prints.the design in the project plan design, preliminary design and construction drawing design, and construction units can take advantage of and design company has dozens of times and can make use of BIM technology of drawing review.

In addition, the construction unit can use BIM technology to arrange funds reasonably and review the payment of the progress payment.

Especially for design changes, the project cost can be adjusted quickly, and relevant components are related to facilitate settlement.

Construction unit can use BIM model to press the time, according to the process, according to the area project cost, convenient cost control, make the detailed management, such as control material dosage, reasonably determine the material price

\section{Settlement stage}

There are several areas where problems can arise in settlement

When the construction contract and the on-site visa understanding are in and out, unilaterally make the explanation favorable to the construction party, the understanding error occurred.

(b) Due to lack of investigation and reliable first hand data, the budget quota, meter or supplementary quota is not reasonable, so the actual cost is far from the norm.

(c) In order to obtain more income unilaterally, some construction units have overestimated the cost by using multiple project quantities and high set quotas

(d) Due to the uneven level of engineering cost staff, the settlement becomes unreal

The accuracy of BIM model ensures the fast and accurate settlement, and most of the checking work is completed during the construction phase, which reduces the wrangling between the two sides and speeds up the settlement speed. Bim makes it in the construction project cost management informationization has incomparable advantage, to enhance the level of construction project cost management information and improve efficiency, and improve the cost management process, has positive significance

As the BIM technology application in the cost management, the construction industry will become more transparent, more orderly, and relevant enterprises can earn a reasonable profit, the center of gravity will also tend to the development of the enterprise internal management, cost control, technical innovation, etc

\section{References}

[1] xuecai Han. Application analysis of BIM in engineering cost management, construction technology [J], 2014.9

[2] guanpei He. BIM general [M].Beijing: China construction industry press, 2017 